\title{
CONCEPCIONES DOCENTES ACERCA DE LOS ERRORES QUE COMETEN LOS ESTUDIANTES AL RESOLVER OPERACIONES BÁSICAS CON FRACCIONES
}

\author{
Jeser C. Candray \\ jeser.candray@unesp.br,jccandray@hotmail.com \\ https://orcid.org/0000-0003-3793-8564 \\ Universidad Francisco Gavidia \\ Instituto de Ciencia, Tecnología e Innovación \\ El Salvador
}

Recibido: 21 de enero de 2021 Aceptado: 25 de mayo de 2021

\begin{abstract}
Resumen
El objetivo de este artículo fue describir las concepciones docentes acerca de los errores que cometen los estudiantes al resolver operaciones básicas con fracciones. Fueron examinadas las concepciones de los docentes acerca de qué es error, causas del error, operación básica con fracciones más fácil y más difícil de resolver por los estudiantes, tipos de errores que comenten los estudiantes al resolver operaciones básicas con fracciones y estrategias para la enseñanza y el tratamiento de los errores. El estudio se realizó a partir de un cuestionario digital aplicando el criterio de red y el análisis se hizo a través de la interpretación de resultados estadísticos y por medio del análisis de contenido. Los resultados principales mostraron una concepción docente negativa acerca del papel del error en el aprendizaje de la matemática, categorías de errores por operación básica y algunas predisposiciones a metodologías tradicionales de la enseñanza de la matemática.
\end{abstract}

Palabras clave: Análisis de contenido, análisis de errores, fracciones.

\section{TEACHERS' CONCEPTIONS OF THE ERRORS COMMITED BY STUDENTS WHEN RESOLVING ELEMENTARY OPERATIONS WITH FRACTIONS}

\begin{abstract}
The aim of this article was to describe the teachers' conceptions of the errors committed by students when resolving elementary operations with fractions. This article studied the teachers' conceptions of what is a errors, the causes of the errors, which are easier or difficult elementary operations for the students, which are the types of errors committed by the students when resolving elementary operations with fractions, and strategies for the teaching and the treatment of the erros. The study was carried out from a digital questionnaire, applying the network criterion and the analysis was carried out through the interpretation of the statistical results and the analysis of the content. The main results show a negative teacher's conception of the role of the errors in the learning of mathematic, errors categories for elementary operations and some predisposition towards traditional methodologies of teaching mathematics.
\end{abstract}

Key words: Content analysis, Analysis of errors, fractions. 


\section{CONCEPÇÕES DOS DOCENTES SOBRE OS ERROS DOS ESTUDANTES QUANDO RESOLVEM OPERAÇÕES BÁSICAS COM FRACÇÕES}

\section{Resumo}

O objetivo deste artigo foi descrever as concepções dos docentes sobre os erros que os alunos cometem ao resolver operações básicas com frações. Primeiramente, foram examinadas as concepções dos docentes sobre o que é erro, causas do erro, operação básica com frações mais fácil e mais difícil de resolver pelos alunos, tipos de erros que os alunos cometem ao resolver operações básicas com frações e estratégias de ensino e manejo de erros. O estudo foi realizado a partir de um questionário digital aplicando o critério de rede e a análise foi feita por meio da interpretação dos resultados estatísticos e da análise de conteúdo. Os principais resultados mostraram uma concepção de ensino negativa sobre o papel do erro na aprendizagem da matemática, categorias de erros por operação básica e algumas predisposições às metodologias tradicionais de ensino da matemática.

Palavras-chave: Análise de conteúdo, análise de erros, frações.

\section{Introducción}

La importancia de conocer y estudiar las concepciones acerca del error que cometen los estudiantes al hacer matemática está en el presupuesto que esta al estudiarlos se pueden tomar acciones en el proceso educativo (Ponte, 1992). Además, es a partir de las ideas que tiene el profesor sobre qué es matemática, cómo se hace matemática, qué es error y cuál es el papel que juega en el proceso de aprendizaje y enseñanza que se diseñarán estrategias para su tratamiento (Muniz, 2017). En esa misma línea, Ponte (1992,) señala que las concepciones condicionan la forma en que se abordarán las tareas.

Para Ponte las concepciones en general juegan una especie de filtro, por un lado, son "indispensables porque estructuran el sentido que damos a las cosas (y) por otro lado, actúan como elemento bloqueador en relación a las nuevas realidades o a ciertos problemas, limitando nuestras posibilidades de actuación y comprensión" (Ponte, 1992, p. 1). Añade el autor que estas concepciones se forman a partir de un proceso simultáneo entre nuestras experiencias individuales y el contraste con los demás.

En este sentido, este texto pretende describir las concepciones docentes sobre los errores de los estudiantes de Educación Básica al resolver operaciones básicas con fracciones (OBF). Así, se busca describir las ideas que tiene el profesorado sobre el trabajo de los estudiantes al enfrentarse a la resolución de problemas y ejercicios con las operaciones suma, resta, multiplicación y división de fracciones y cómo estas concepciones, construidas a lo largo de su experiencia profesional, inciden en la metodología para la enseñanza de estos contenidos. 
Para el abordaje de estas concepciones docentes se propone un análisis a partir de cinco grandes unidades de estudio derivadas del objetivo principal: ¿qué es error en matemática para los docentes?; ¿cuáles creen que son las causas de ese error?; ¿cuál creen que es la operación básica más fácil y la operación básica más difícil de resolver por los estudiantes?; ¿qué tipo de errores han detectado que cometen los estudiantes al resolver OBF? y ¿qué estrategias utilizan los docentes para enseñar las OBF?

Antes de presentar los resultados de la consulta, se considera necesario traer a discusión algunas ideas acerca de unos conceptos e ideas que serán tratadas a partir de las palabras de los docentes. Es claro que no se pretende agotar el tema.

Se podría hablar de error en Educación Matemática "cuando un alumno proporciona una respuesta incorrecta a una cuestión matemática que se le plantea se puede decir que su respuesta es errónea, y la solución proporcionada es un error en relación con la cuestión propuesta" (Rico, 1995, p. 6). Además, el error puede ser considerado "como la presencia en el alumno de un esquema cognitivo inadecuado y no solamente como consecuencia de una falta específica de conocimiento o de un despiste" (Socas, 1997, p. 1). Socas relaciona el error con dificultad siendo el error una manifestación de las dificultades que tienen los estudiantes. Esto suele ser importante, dado que puede existir una confusión entre ambos términos y de estos con la idea de obstáculo ${ }^{1}$.

Ahora bien, ¿cómo se percibe el error en contextos escolares (y no escolares)? desde una visión tradicional, el error tiene un status negativo. A nadie le gusta errar. Según Astolfi (2003), el error tiene distintas connotaciones; es visto como un fallo del sistema, como un vacío, un descuido o desinterés del que aprende y como disparates del estudiante. Todas estas ideas en torno al error refuerzan el papel negativo en el aula de matemática. ¿Qué suele ser señalado como error en un examen de matemática (o en cualquier otro)?, ¿Qué se corrige en una exposición?, ¿Qué pasa cuando el estudiante es invitado a resolver un problema en la pizarra? Todas estas situaciones hacen que el error no sea bienvenido en el aula, que sea mal visto errar y que errar en definitiva trae consecuencias en la vida escolar.

\footnotetext{
${ }^{1}$ Para una discusión más específica de este tema se recomienda consultar: Brousseau G. (1983) Les obstacles épistémologiques el les problèmes. En mathématiques. Recherches en Didactique des Mathématiques, Vol.4.núm. 2, pp.165-198; Bachelard, G. (1938) La formation de l'esprit Scientifique. Vrin, París. 1980. Traducción al catellano, 1985. La formación del espíritu Científico. Siglo Veintiuno. México y; Tall, D. (1989). Different Cognitive Obstacles in a Technological Paradigm. Research Agenda for Mathematics Education. Research Issues in The Learning and Teaching for Algebra. Wagner-Kieran Editors. N.C.T.M.
} 
Sin embargo, Astolfi afirma que desde posiciones constructivistas se aboga por considerar el error desde una visión más positiva: “en los modelos constructivistas los errores no se consideran faltas condenables ni fallos de programa lamentables: son síntomas interesantes de los obstáculos con los que se enfrenta el pensamiento de los alumnos" (Astolfi, 2003, p. 14) y añade que el error está en el centro del proceso de aprendizaje y que adquiere un nuevo estatus: "el de indicador y analizador de los procesos intelectuales" (Astolfi, 2003, p. 15). De este modo, será interesante abordar las posturas del docente ante el concepto del error en el cuestionario.

Por otro lado, ¿qué causa el error? Desde posiciones más tradicionales, las causas del error se encuentran en el estudiante ya sea por descuidos o desinterés o en el docente por la metodología utilizada que no dan una visión general y correcta de la temática estudiada. Es decir, en esta visión, el error es evitable si se cuenta con interés del estudiante, si se cuenta con una buena metodología de enseñanza y un profesor que la ejecute (Astolfi, 2003). Sin embargo, en posiciones constructivistas se asume que el error es parte del aprendizaje y que es independiente del ánimo del estudiante y de la metodología que utilice el profesor. Para Astolfi (2003) “el error es una dificultad objetiva en la apropiación del contenido enseñado" (p. 21). Cury (2017), por su parte, afirma que asumir que el error es parte del aprendizaje pasa por considerar que el análisis del trabajo de los estudiantes debe moverse del producto al proceso, es decir, a los procedimientos matemáticos que ellos realizan. Esta situación permite "la posibilidad de que los errores cometidos vengan a ser discutidos y puedan ser fuente de nuevos aprendizajes" (Cury, 2017, p. 38). De hecho, Cury, citando a Borasi (1985), ofrece una interpretación interesante de los errores ya que para ella estos son "trampolines del aprendizaje", Cury, (2017, p. 39).

Estas dos concepciones acerca de qué es error y sus causas condicionan las estrategias que se tomarán para su tratamiento. Tradicionalmente en el aula el error es perseguido, dando lugar a lo que Astolfi (2003) llama "síndrome del rotulador rojo", es decir, la postura del docente de encontrarlos y señalarlos. Así, desde esta visión, para el tratamiento del error, suele utilizarse palabras como refuerzo, retroalimentación, actividades complementarias que buscan afianzar, fijar o asegurar el conocimiento enseñado. Para ello es común utilizar las guías de trabajo con ejercicios y/o problemas con o sin distintos niveles de dificultad y repetir conceptos o procesos para desvanecerlos. 
A su vez, Abrate, Pochulu y Vargas (2006), asumiendo una posición de no recomendar estrategias universalmente aplicables, consideran que existen estrategias más o menos adecuadas acorde a cada situación. Por ejemplo, se asume que la corrección sistemática no hace que el error desaparezca y se considera que para su tratamiento se deben crear estrategias en el aula para que sean los mismos estudiantes quienes perciban los errores y que su conocimiento sea validado por ellos mismos.

Martin Socas (1997) amplía esta idea y propone que las estrategias de tratamiento del error pueden comenzar desde la enseñanza del tema, y esta enseñanza debe

ir encaminada a detectar los errores y provocar el conflicto en los alumnos, fomentando ideas que permanezcan activas más allá de la clase de Matemáticas y capacitándole para evaluar si sus ideas o métodos son o no correctos en una determinada tarea matemática. (Socas, 1997, p. 35).

Se percibe en esas ideas que el tratamiento del error va más allá de la corrección y no implica que para su presumible corrección se necesite de una metodología preventiva, sino un abordaje diversificado. Nuevamente, será interesante conocer y analizar las posturas del docente ante las estrategias planteadas para el tratamiento del error en las OBF.

\section{Cuestiones Metodológicas}

La investigación de este estudio es de tipo cualitativo, considerando para este estudio no establecer una hipótesis a priori, y se asume una posición no neutral de parte del investigador, sin intención de establecer verdades ad eternum y no pretende generalizar sus conclusiones (Garnica, 2004) y para la recopilación de la información se hizo por medio de un cuestionario digital con preguntas de opción múltiple y preguntas abiertas.

Para la selección de los participantes del estudio se buscó que cumplieran los siguientes requisitos: que fueran docentes formados en matemática, que enseñaran matemática en el Tercer Ciclo de Educación Básica en el año 2020 (estudiantes entre 12 y 15 años de edad), que tuvieran al menos tres años de ejercer la docencia, que su grupo de estudiantes fuera del departamento de San Salvador, que tuvieran la disposición y capacidad de responder un cuestionario digital y que aceptaran el uso de la información para actividades académicas. A los participantes se les garantizó su anonimato.

La selección de la muestra para este estudio es no probabilista, intencional (Creswell, 2010) y con criterio de red. Es no probabilista porque no busca generalizar los resultados, es 
intencional porque los participantes fueron seleccionados con criterios de proximidad y con criterio de red porque se pidió que el cuestionario fuese compartido por otros colegas docentes de matemática (Sampieri, Collado y Lucio, 2010). Además de los criterios anteriores se propuso que este cuestionario fuese respondido por al menos veinticinco docentes.

El instrumento para la obtención de la información es un cuestionario cerrado, diseñado en la aplicación de Google Forms. El uso de este instrumento se ha popularizada recientemente debido a la crisis generada por el Covid 19 que impide el acceso y contacto entre personas. Además, se buscó que el diseño del cuestionario cumpliera los criterios siguientes: veinte preguntas abiertas y cerradas, que sea respondido en un tiempo máximo de treinta minutos. Para garantizar estos criterios, el cuestionario fue compartido a cinco docentes de matemática, que no participarían del estudio final, quienes devolvieron el cuestionario con sus comentarios, observaciones y el tiempo utilizado.

El cuestionario estaba dividido en tres partes. La primera parte se incluyeron preguntas de información profesional y laboral para poder verificar el cumplimiento de las condiciones previamente establecidas, la segunda parte constaba de preguntas preferentemente cerradas y la tercera parte constaba de preguntas abiertas que buscaban ampliar las respuestas obtenidas de la parte dos. Una vez integradas las observaciones, se compartió con docentes y se pidió que se compartiera con otros docentes. El instrumento estuvo disponible para el llenado en un plazo de dos semanas. Las respuestas de los docentes, dado que se utilizó la aplicación Google Forms, era almacenada directamente en la aplicación y para su tratamiento fue extraída la información por medio de una hoja de Excel.

El análisis de los resultados se hizo en dos vías. Primero, se utilizaron como apoyo los datos estadísticos de los cuestionarios de las preguntas que tenían respuesta de opción múltiple. El uso de datos cuantitativos en estudios cualitativos está aceptado, no obstante, un investigador cualitativo no busca quedarse únicamente con el valor numérico, sino que busca comprender qué implicaciones tiene este valor para la comprensión del problema de estudio (Bogan y Biklen, 1994, p. 195). Así, esta información se presenta por medio de gráficos y tablas estadísticas. Además, para el análisis de las preguntas abiertas se hace uso del análisis de contenido (AdC) inspirados en los presupuestos de Moraes (1999). Para cada una de las respuestas cualitativas se creaba un corpus, que incluía todas las respuestas de los docentes participantes; luego se procedió a la unitarización de las respuestas, que consistía en desglosar del corpus las respuestas 
de los docentes en frases u oraciones que constituían una idea unitaria, esta idea unitaria se buscaba fuese lo más simple posible evitando que contase con otros temas o ideas en ella y; por último, se categorizaron las respuestas, es decir, cada una de las frases u oraciones eran agrupadas según adjetivos o palabras clave que generaban categorías emergentes.

Para el cierre del plazo del llenado del instrumento se contó con 46 cuestionarios completados de los cuales 34 cumplieron los requisitos establecidos. Además, se verificó que cada participante respondiera una sola vez el cuestionario. Sobre la experiencia docente, 26\% dijo tener entre 3 y 10 años de experiencia, 29\% entre 11 y 15 años de experiencia, 23\% entre 16 y 20 años de experiencia y el 20\% dijo tener más de 20 años de experiencia. También se consultó sobre el nivel de formación docente, del total de participantes el 53\% tiene formación de profesorado, $44 \%$ a nivel de licenciatura y un 3\% dijo contar con maestría. Según el lugar de trabajo cerca de $90 \%$ labora en el sector público, un 6\% dijo laborar en el sector privado y otro $6 \%$ dijo trabajar en ambos sectores.

Por último, se les consultó los niveles en que imparten clases: uno de cada tres trabaja exclusivamente en el Tercer Ciclo de Educación Básica; uno de cada cuatro imparte clases en Tercer Ciclo y en Bachillerato; uno de cada cuatro imparte clases en Segundo y Tercer Ciclo de Educación Básica y; el resto en todos los niveles educativos.

\section{Resultados del Estudio}

A continuación, se presentan los resultados de la investigación según las unidades de estudio: ¿qué es error?; causas del error; operación básica más fácil y más difícil; categorías de errores y; estrategias docentes para tratar el error. Las respuestas de los docentes son presentadas entrecomilladas.

\section{¿Qué es el error según las concepciones docentes?}

Como se estableció en las ideas iniciales, caracterizar las concepciones docentes acerca del error en el proceso de aprendizaje de los estudiantes puede dar luz sobre cómo enseñan matemática y cómo consideran que este se debe tratar en el aula. Ante la pregunta ¿qué entiende por error en el proceso de aprendizaje de los estudiantes? los docentes dieron una diversidad de respuestas que al analizarlas por medio del $\mathrm{AdC}$ se pudo construir nueve categorías emergentes, que se presentan en el cuadro siguiente (ver Cuadro 1). 
Cuadro 1. Categorización: ¿qué es error?

\begin{tabular}{|c|c|}
\hline Pregunta & Categorías \\
\hline \multirow{3}{*}{ Según su criterio y } & Aplicación incorrecta \\
\cline { 2 - 3 } experiencia, ¿qué & Equivocación \\
\cline { 2 - 3 } entiende por error & Procedimiento no lógico \\
\cline { 2 - 2 } en el proceso de & No asimilación del estudiante \\
\cline { 2 - 2 } aprendizaje de los & No adquisición de una competencia del estudiante \\
\cline { 2 - 3 } estudiantes? & Conocimiento incorrecto \\
\cline { 2 - 3 } & Aprendizaje incorrecto \\
\cline { 2 - 3 } & Olvido \\
\cline { 2 - 3 } & Dificultades \\
\hline
\end{tabular}

Fuente: Elaboración propia.

Las concepciones docentes acerca del error en el trabajo de sus estudiantes reflejan una valoración negativa sobre el mismo, esto se deriva de las respuestas de los docentes al asociar el error con lo incorrecto, el olvido, la dificultad o con la equivocación. Los docentes al mencionar olvido se refieren a que el estudiante aprende momentáneamente un contenido o procedimiento, pero que luego es olvidado y la asociación del error con la dificultad se debe a que consideran que estas aparecen por las complicaciones propias del tema que está aprendiendo el estudiante. Esta mención a las dificultades de los estudiantes va en línea a lo señalado por Soacas (1997). Además, los docentes consideran que el error aparece en distintos estadios que discutimos a continuación.

Primero, este se verifica en aspectos cognitivos tales como lo evidencian las categorías no asimilación, no adquisición y aprendizaje incorrecto, es decir, el estudiante, en esa lógica, no logra interiorizar el conocimiento, lo que genera una dificultad para realizar las actividades matemáticas. Por otro lado, también existen categorías que hacen referencia a aspectos propiamente procedimentales (Rico, 1995), como lo dejan ver las categorías aplicación incorrecta, equivocación y procedimiento no lógico; tales categorías refuerzan la idea que el error está presente o puede verificarse cuando el estudiante trabaja y muestra sus procedimientos al resolver ejercicios o problemas matemáticos. 
Se podría decir que según estas concepciones docentes el error es una aplicación incorrecta de un procedimiento matemático que puede ser causado por la no asimilación de un conocimiento previo. En lecturas más específicas de este punto, un docente señala el papel de los conocimientos previos cuando dice que el error aparece porque "no se realizó el proceso correcto de interiorización conscientemente del algoritmo matemático" o "cuando el estudiante no adquiere las competencias necesarias de un tema específico”. Algunos los docentes presentan posturas autocríticas y no apuntan solo al trabajo del estudiante, sino también al rol docente al afirmar que el error es producto de "un aprendizaje incorrecto por parte del alumno y mala metodología por parte del maestro" que ya remite a causas de esos errores.

De estas ideas de los docentes, se puede verificar una visión tradicional del error, en especial la última afirmación del docente que relaciona el error a una falla del sistema o de la metodología de enseñanza; en consecuencia, esto robustece la idea que el error puede ser evitado por medio de una metodología idónea que es responsabilidad del docente, generando un sentimiento de culpa en el profesorado al constatar una y otra vez la aparición de los mismos (Imbernón, 2001), (Abrate, Puchulu y Vargas, 2006).

En esta línea, ¿cómo podría pasarse de esta concepción negativa acerca del error a posiciones más propositivas como las de Astolfi (2003) y Cury (2017)? Se podría decir que este movimiento podría darse a partir de un cambio de enfoque de resultados a los procedimientos de los estudiantes, tal como es planteado por Cury. En procesos formativos-reflexivos podría tratarse con docentes acerca de estatus natural que tiene el error en la ciencia. En estos procesos formativos podría discutirse algunas afirmaciones acerca del error planteadas por estudiosos del tema tales como, "el niño puede no haber errado, y sí haber resuelto otro problema" de Anna Franchi (Cury, 2017, p. 12), “el error como un conocimiento", "el error creativo" Astolfi (2003), "los errores son hipótesis legitimas basadas en concepciones y creencias adquiridas a lo largo de la vida" de Antonio José Lopes (Cury, 2017, p. 13), entre otras. Todas estas ideas desmontan mitos acerca del error y sus consecuencias en el proceso educativo general y específico en matemática.

\section{Causas del error, según los docentes}

Otro punto de interés, es profundizar acerca de las causas de esos errores. Para ello, se abordaron dichas concepciones por medio de dos preguntas complementarias en el cuestionario. En una primera pregunta, se consultó a los docentes por medio de un ítem cerrado de opción 
múltiple contando con seis opciones posibles pudiendo marcar más de una opción. La pregunta fue: ¿por qué los estudiantes erran al resolver OBF? Las respuestas obtenidas fueron las siguientes (Tabla 1).

Tabla 1. Según su criterio y experiencia, ¿Por qué los estudiantes erran al resolver OBF?

\begin{tabular}{lll}
\hline Opciones & Total & Porcentaje \\
\hline Desinterés por aprender de parte del estudiante & 20 & $57,14 \%$ \\
Por distracción/descuido del estudiante & 8 & $22,86 \%$ \\
Porque se enseña mal en grados anteriores & 19 & $54,29 \%$ \\
Porque el profesor/a no muestra interés en enseñar & 3 & $8,57 \%$ \\
Por la propia dificultad del aprendizaje de las & 16 & $45,71 \%$ \\
fracciones & 9 & $25,71 \%$ \\
Porque se dedica poco tiempo para los temas & 34 & \\
Total docentes & & \\
\hline
\end{tabular}

Fuente: Elaboración propia de acuerdo al formulario docente.

La interpretación de estos resultados puede tener distintas aristas, sin embargo, las seis opciones fueron construidas para analizar tres agentes distintos: el estudiante, el docente y el currículo. Estas categorías pueden ser analizadas en conjunto o separadamente. De forma global, se puede decir que casi dos tercios de los docentes ven en el desinterés de los estudiantes y en la mala enseñanza del tema en años anteriores las principales causas del error. La razón menos seleccionada fue la propia actividad pedagógica del docente. Un poco menos de la mitad de los docentes consideró que la propia dificultad del contenido es la causa principal del error de los estudiantes. Llama la atención como los docentes tienden a señalar a sus colegas docentes de años anteriores y a los propios estudiantes como agentes causantes del error.

Ahora bien, para profundizar en el análisis, se preguntó a los docentes acerca de ¿Cuál es la causa que hace que los estudiantes erren al resolver operaciones básicas con fracciones? Las respuestas fueron tratadas por medio del AdC, construyéndose categorías emergentes que se presentan en el cuadro siguiente (Cuadro 2): 


\section{Cuadro 2. Categorización: causas del error}

\begin{tabular}{lll}
\hline Pregunta & Agente & Categoría \\
\hline & & Olvido \\
& Falta de interés del estudiante \\
\multirow{2}{*}{$\begin{array}{l}\text { Según su criterio y experiencia, } \\
\text { ¿Cuál es la causa que hace que }\end{array}$} & Estudiantes & Predisposición emocional \\
los estudiantes erren al resolver & & Fo comprenden la teoría ni la práctica \\
operaciones básicas con & & Falta de práctica \\
fracciones? & & Conocimientos previos \\
& & No seguir procedimientos \\
\cline { 2 - 3 } & Docentes & Docentes de años anteriores \\
& & Enseñanza incorrecta \\
\hline & Currículo & Cuestiones curriculares \\
\hline
\end{tabular}

\section{Fuente: Elaboración propia}

Las respuestas hacen más énfasis en las distintas actitudes que los estudiantes toman ante el proceso educativo que generan, según ellos mismos, errores. Las actitudes mencionadas son el olvido, la falta de interés del estudiante, falta de atención como propiciadores del error. Otro de los docentes señaló que existe una "falta de interés del alumno en aprender correctamente el proceso" lo que pude llegar a generar que los estudiantes olviden lo aprendido.

Otra categoría construida a partir de los aportes de los docentes es la predisposición emocional, que puede entenderse como una aversión de los estudiantes a la matemática. Esta aversión surge de su proceso de escolarización que hace que el estudiante rechace a priori efectuar procesos matemáticos al considerarlos difíciles o complejos incluso antes de efectuarlos. Otra categoría por los docentes es la falta de conocimientos teóricos y prácticos acerca del asunto, lo que es comúnmente llamado de conocimientos previos. Estos conocimientos previos se vinculan con una categoría que tiene al docente como agente principal, es decir, la categoría docente de años anteriores que hace referencia a que gran parte de los errores de los estudiantes es causado por la forma en que los docentes enseñan el contenido. En este sentido un docente señalaba que: "docentes no especialistas -no especialistas en matemática- colocan ejercicios demasiados engorrosos a los alumnos" y esto causa los errores de los estudiantes. 
Por último, referente al agente currículo y en la categoría cuestiones curriculares, un docente señalaba que "no se acostumbra a enseñar las operaciones con fracciones, siguiendo una secuencia lógica y ordenada y no se contextualiza la operación con situaciones de la realidad. Para identificar la verdadera naturaleza de los algoritmos a utilizar”. Es decir, algunos docentes señalan que la multiplicidad de métodos de resolución en matemática puede generar confusión en los estudiantes y esto los lleva a errar.

Ahora bien, si hacemos un análisis comparativo de las causas de los errores a partir de las concepciones docentes, se puede decir que se perciben dos causas relacionadas a lo que se conoce como obstáculos epistemológicos y obstáculos didácticos. Las categorías docentes de años anteriores y enseñanza incorrecta son comparables con los obstáculos didácticos y los obstáculos epistemológicos, por ser obstáculos relacionados a la asimilación del que aprende, pueden relacionarse a las categorías no comprenden la teoría ni la práctica y a la falta de conocimientos previos.

No obstante, de estas concepciones docentes acerca del origen del error en el proceso de aprendizaje; se puede verificar la existencia de una carga negativa de los docentes acerca del error, es decir, el error es visto como un elemento ajeno, una anormalidad dentro del proceso de aprendizaje y no como algo natural. Esta connotación lleva entonces a que el error sea perseguido, señalado y busque ser eliminado y no como un indicador del proceso de aprendizaje que permita ver el error como un trampolín para el aprendizaje como lo señala Borasi (Cury, 2017).

\section{Operación básica con fracciones más fácil / más difícil de resolver, según los docentes}

Al consultarle a los docentes acerca de cuál es la OBF más fácil y cuál es la más difícil de resolver para los estudiantes, los resultados fueron los siguientes (Tabla 2).

Tabla 2. Operación más fácil y difícil según criterio y experiencia docente.

\begin{tabular}{lll}
\hline Operación & Fácil & Difícil \\
\hline Suma & $14.7 \%$ & $47.1 \%$ \\
Resta & 0 & $32.4 \%$ \\
Multiplicación & $79.4 \%$ & $2.9 \%$ \\
División & $5.9 \%$ & $17.6 \%$ \\
Total & $100 \%$ & $100 \%$ \\
\hline
\end{tabular}

Fuente: Elaboración propia de acuerdo al formulario docente. 
Al estudiar las respuestas de los docentes se puede verificar que casi 4 de cada 5 docentes consultados consideran que la multiplicación es la OBF más fácil de resolver por los estudiantes, siendo este un resultado contundente. El resto de OBF aparecen con números muy dispersos. Al intentar profundizar, en una pregunta posterior, acerca de porqué los docentes consideraban a la operación multiplicación como la más fácil de resolver, las justificaciones a esta idea de los docentes era que esta se considera un "proceso lineal, sencillo de resolver y que requiere, en su mayoría, un único algoritmo". Así, un docente argumentó su respuesta afirmando que "La regla básica para multiplicar fracciones es relativamente sencilla porque deben multiplicarse los numeradores entre sí y los denominadores entre sí”. Otras justificaciones acerca de esta concepción es que consideran que la multiplicación es fácil de memorizar por los estudiantes y que es fácilmente asociada a la suma.

Los docentes que consideran que la suma es la operación más fácil argumentaban que esta es así “cuando [las fracciones] son homogéneas” y los docentes que señalan a la división como la operación más fácil se entiende que se debe a que "cuando hacen el cambio en la segunda fracción para hacerla multiplicación casi siempre la dejan igual”. El total de categorías asociadas a la pregunta “operación básica con fracciones más fácil”, según los docentes, está en el cuadro 3.

\section{Cuadro 3.}

Por favor explique, ¿por qué considera que esa operación básica con fracciones que señaló en la pregunta anterior resulta más fácil de resolver por los estudiantes?

\section{Operación Categoría}

\begin{tabular}{ll}
\hline & $\begin{array}{l}\text { Multiplicación lineal } \\
\text { Sencillez del proceso } \\
\text { Multiplicación }\end{array}$ \\
& Un solo algoritmo \\
& Por memorización \\
& Se asocia con la suma \\
Suma & Sumas homogéneas \\
\hline Resta & Facilidad del procedimiento \\
\hline División & División/Multiplicación \\
\hline Fuente: Elaboración propia. & \\
\hline
\end{tabular}


La contundencia de los resultados acerca de la OBF que es la más fácil de resolver por los estudiantes, contrasta con los resultados acerca de cuál es la OBF más difícil de resolver por los estudiantes. Los datos reflejan que cerca del 50\% de los docentes consideraron que la operación suma es la más difícil de resolver por los estudiantes; un 32\% se refirió a la operación resta, el 17\% a la división y la multiplicación menos del 3\% la consideró como la más difícil de resolver por los estudiantes. El hecho que la suma y resta destaquen como las OBF más difíciles de resolver puede deberse a que ambas tienen procesos algorítmicos similares; de igual forma, el hecho que la multiplicación sea la OBF menos puntuada como difícil es entendible debido a que esta operación es considerada por la mayoría de los docentes consultados como la más fácil.

Profundizando con los docentes el por qué consideran que la suma es la OBF más difícil, ellos justificaron su respuesta por la dificultad del proceso y de los algoritmos, operaciones combinadas, los denominadores heterogéneos, los métodos de enseñanza, el MCM y los conocimientos previos. Ellos señalaron que la dificultad en los procesos algorítmicos en la suma de fracciones es debido a que "implica un proceso que no es parecido a la suma de números enteros" y "requiere la combinación de otras operaciones y además aprenderse el procedimiento". Esto es más notorio cuando las fracciones son heterogéneas, así un docente afirma que en ese caso "siempre se vuelve complicado para el estudiante [...], ya que se requiere la utilización de diferentes conocimientos previos como: mínimo común múltiplo y la homogenización de las fracciones" entre otros.

En sintonía con estas ideas respecto de la suma, para la operación resta se hace referencia a estas mismas razones solo que a esta se añade la categoría ley de signos como argumento para justificar su decisión. Así un docente afirma que "La ley de los signos agrega a la resta una mayor dificultad que la suma". Por último, una de las razones por las cuales los docentes creen que la división es la OBF que más se les complica a los estudiantes se debe a que ellos "olvidan que es producto en cruz" o bien porque "confunden el procedimiento con el de la multiplicación". El total de estas categorías, de acuerdo al tipo de OBF, está presente en el Cuadro 4 a continuación: 


\section{Cuadro 4.}

Por favor explique, ¿por qué considera que esa operación básica con fracciones que señaló en la pregunta anterior resulta más difícil de resolver por los estudiantes?
Operación
Categorías

\begin{tabular}{|c|c|}
\hline \multirow{7}{*}{ Suma } & Dificultad del proceso y de los algoritmos \\
\hline & Operaciones combinadas \\
\hline & Confunde con multiplicación \\
\hline & Método de enseñanza del docente \\
\hline & Conocimientos previos \\
\hline & Denominadores heterogéneos \\
\hline & Por MCM \\
\hline \multirow{5}{*}{ Resta } & Procesos y algoritmos \\
\hline & Ley de los signos \\
\hline & Método de enseñanza \\
\hline & Denominadores heterogéneos \\
\hline & Por el MCM \\
\hline \multirow{4}{*}{ División } & Resuelven como multiplicación \\
\hline & Olvidan procesos \\
\hline & Simplificar \\
\hline & Procesos afectivos \\
\hline Multiplicación & Olvidan procesos \\
\hline
\end{tabular}

Fuente: Elaboración propia

\section{Categorías de errores por operación básica con fracciones}

En esta sección se presentan las categorizaciones de los errores que comenten los estudiantes al resolver las OBF que han sido percibidos por los docentes. El proceso seguido para llegar a estas categorizaciones es el mismo empleado con el ejercicio de las unidades de estudio anteriores.

Categorías de errores al operar la suma de fracciones

Al consultarle a los docentes acerca de los errores más comunes que han identificado que cometen los estudiantes al resolver sumas de fracciones se encontraron ocho categorías que se presentan en el Cuadro 5. 
Cuadro 5. Categoría de errores, según docentes, al operar la suma de fracciones

\begin{tabular}{|l|}
\hline Suman fracciones de forma lineal \\
\hline Suman el denominador de fracción Homogénea \\
\hline Identifican mal el denominador \\
\hline No encuentran el MCM \\
\hline Aplicación incorrecta del MCM \\
\hline No dominio del algoritmo \\
\hline Olvidan procedimiento \\
\hline No simplifican fracciones \\
\hline
\end{tabular}

\section{Fuente: Elaboración propia}

La suma de fracciones de forma lineal o suma horizontal de fracciones es la que más fue mencionada por los docentes y se da cuando "los estudiantes tienden a querer sumar numerador con numerador y denominador con denominador"; este error es también conocido como una sobregeneralización de las propiedades de la suma de números enteros sobre la suma de números fraccionarios (Cury, 2017). Un caso particular de esta sobregeneralización se da cuando el estudiante suma el denominador de las fracciones homogéneas, es decir, ese error se da cuando los estudiantes siguen el mismo procedimiento para fracciones homogéneas y heterogéneas. El trabajo con los denominadores es otro error común detectado por un grupo de consultados los docentes. Esta sobregeneralización no es un asunto exclusivo de estudiantes salvadoreños. Lopes (2008) en su artículo destaca este fenómeno y ofrece como alternativa, a partir de diálogos con Peter Hilton, un aprovechamiento didáctico del error considerando casos en los que esta sobregeneralización es verdadera.

Otro error observado por los docentes radica en el cálculo del Mínimo Común Múltiplo (MCM). El uso del MCM para la resolución de fracciones es un método habitual en las aulas de matemática, al menos en El Salvador. Los docentes consultados señalan un doble error a partir de este proceso: primero es que no pueden deducirlo y el segundo que, si lo deducen, no saben aplicarlo. Esto es explicado por un docente participante cuando afirma que los estudiantes "no recuerdan o aplican mal el MCM al establecer el denominador común en una suma de fracciones". Otro error común sucede porque el estudiante olvida o no domina bien el algoritmo de la suma de fracciones, de este modo algunos de los docentes perciben que a los estudiantes 
"se les dificulta recordar en qué orden se realiza el algoritmo". Por último, otros docentes señalan que un error muy común de los estudiantes se da en la simplificación de las fracciones.

\section{Categorías de errores al operar la resta de fracciones}

Son seis las categorías que surgieron a partir de la pregunta ¿cuáles son los errores más comunes que usted ha verificado cuando un estudiante resuelve una resta de fracciones? Las categorías se presentan en el cuadro $\mathrm{F}$ a continuación:

Cuadro 6. Categoría de errores, según docentes, al operar la resta de fracciones

\begin{tabular}{|l|}
\hline Restan de forma lineal \\
\hline Error en el trabajo de los signos \\
\hline Trabajo con el denominador \\
\hline No encuentran el MCM \\
\hline Falta de dominio del algoritmo \\
\hline Los mismos que la suma \\
\hline
\end{tabular}

\section{Fuente: Elaboración propia}

Al igual que en el caso de la suma, la categoría resta de forma lineal es la más mencionada por los docentes y tiene igual interpretación que la operación suma. La categoría error en el trabajo de los signos se debe, según los docentes, a que los estudiantes tienen problemas para tratar con la ley de signos. La categoría trabajo con el denominador, hace referencia a trabajar las fracciones homogéneas y heterogéneas indistintamente y al igual que la categoría falta del dominio del algoritmo son equivalentes a las interpretaciones de los errores en la operación suma. En ese sentido, se puede decir que la categoría que diferencia a la resta en comparación a la suma radica en el trabajo con los signos, dado que ambas siguen el mismo algoritmo tanto para las fracciones homogéneas como con las heterogéneas.

Para Soacas (1997) una forma de tratar los errores producidos por la suma y resta "lineal" podría ser a partir del desarrollo de las fracciones equivalentes, de esa forma el estudiante evita generalizar las propiedades de la suma y resta de enteros para las fracciones.

\section{Categorías de errores al operar la multiplicación de fracciones}

Al consultar a los docentes acerca cuál OBF es la más fácil y cuál la más difícil, se pudo verificar que la multiplicación es considerada por la mayoría de los docentes consultados como la más fácil y la menos difícil para los estudiantes. Pese a esto, las respuestas de los docentes 
reflejan una gran cantidad de errores al momento de trabajar con la multiplicación de fracciones. Las categorías de los errores de los estudiantes al resolver multiplicaciones de fracciones se presentan en Cuadro 7 a continuación.

Cuadro 7. Categoría de errores, según docentes, al operar la multiplicación de fracciones

\begin{tabular}{|l|}
\hline No conocen las tablas \\
\hline Operar con fracciones mixtas \\
\hline Multiplicar fracción con entero \\
\hline Multiplicación cruzada \\
\hline No simplifican las fracciones antes de multiplicar \\
\hline No simplifican al final del proceso \\
\hline Se equivocan en los signos \\
\hline Confunden los números que deben multiplicar \\
\hline Fallan en el algoritmo
\end{tabular}

Fuente: Elaboración propia

Las dos categorías más mencionadas por los docentes es que los estudiantes no conocen las tablas, es decir, que se equivocan en la multiplicación de los números y la multiplicación cruzada, es decir, que multiplican fracciones con "la regla de la división ya que se le enseño a memorizar". Sobre este punto, un docente consultado señala que los estudiantes erran incluso usando la calculadora: "se les olvida las tablas de multiplicación conste si no se les deja usar y si usan calculadora pues la poca experiencia al usarla”.

Dos casos específicos también son mencionados por los docentes. Para ellos los estudiantes erran al multiplicar fracciones que envuelven números enteros y/o números mixtos y añaden que "cuando se multiplica una fracción por un entero [los estudiantes] tienden a multiplicar ambos términos de la fracción por el entero" y el error con el trabajo de las fracciones mixtas se da porque los estudiantes "no convierten a fracciones impropias para multiplicar".

Ahora bien, retomando cuál era el error más común en la operación suma y resta de fracciones que hacía referencia a la operación suma y resta de forma lineal como si fueran números enteros, se puede entender que la operación multiplicación con fracciones sea más fácil de asimilar para los estudiantes, puesto que el procedimiento es ese el seguido para la multiplicación de números enteros. Las otras categorías determinadas a partir de las 
concepciones docentes son que "se equivocan en los signos" y la "no simplifícación de fracciones" ya sea antes del proceso de multiplicar o al final del proceso.

\section{Categorías de errores al operar la división de fracciones.}

Ocho categorías surgieron a partir de las concepciones docentes acerca de los errores de los estudiantes al resolver divisiones de fracciones. Estas categorías se presentan en el Cuadro 8 a continuación.

Cuadro 8. Categoría de errores, según docentes, al operar la división de fracciones

No puede encontrar el recíproco

Trabajan como multiplicación

No simplifican las fracciones

Involucrar conocimientos previos

Se equivocan con los signos

Olvida el algoritmo

Planteamiento de algoritmos

Confunden procesos

Fuente: Elaboración propia

El principal error que los docentes consideran que son cometidos por los estudiantes es determinar el recíproco de una fracción. El uso del recíproco de una fracción es usado cuando se pretende convertir una división de fracciones en una multiplicación, para ello, lo que se hace es multiplicar la fracción dividendo por el recíproco de la fracción divisor. Allí la importancia de encontrar el reciproco en este método. Otra categoría resulta de la confusión de los estudiantes en resolver la división de fracciones como si fueran multiplicaciones.

Categorías como conocimientos previos y error con los signos son categorías que se comparten con la multiplicación, son señaladas por los docentes debido a que los estudiantes "no recuerdan las tablas de multiplicación" y "no aplican correctamente la ley de los signos" y ya han sido descritas anteriormente. La categoría confunde procesos, se refiere a que los estudiantes realizan la división de fracciones haciendo el recíproco de la fracción divisora y luego hacen la multiplicación cruzada. 
Haciendo una comparación de las categorías de errores entre la operación multiplicación y división, se puede verificar que comparten los errores producto del trabajo con números mixtos y la confusión de procesos algorítmicos.

Profundizando acerca de las concepciones docentes acerca del error de los estudiantes cuando resuelven división de fracciones, Lopes $(2008$, p.17) reconoce que la "las posibilidades de un abordaje intuitivo para la división de fracciones son escasas, las aplicaciones realistas son incluso más escasas"; esto queda más aun evidente cuando el enfoque del tratamiento de la división de fracciones se reduce a estudiarla únicamente como la inversa de la multiplicación, cuando la división de fracciones puede ser explorada a partir del razonamiento proporcional y de este modo comprender la división de números enteros como una fracción y la división de fracciones como una resta sucesiva . Esto es tratado por Flores Peñafiel (2014).

Con esto en mente, Lopes (2008, p. 19-20) se apoya en un estudio realizado con niños de 12 años de sexta serie de educación fundamental (sexto grado en El Salvador) por él en que se la división de fracciones es presentada como un problema, sin prescripción de reglas. Este estudio mostró las ventajas de ofrecer esta exploración a los estudiantes y cómo a partir de la introducción de la división como problemas se construyó intuitivamente por los estudiantes la regla para la división de fracciones.

\section{Estrategias docentes para la enseñanza de las fracciones y el tratamiento del error}

Se consultó a los docentes acerca de las estrategias que utilizan para la enseñanza de las fracciones. Las estrategias y los resultados aparecen en la Tabla 3 siguiente.

Tabla 3. Estrategias utilizadas para enseñar OBF

\begin{tabular}{lll}
\hline Estrategia & Sí & No \\
Resolver el ejercicio o problema en la pizarra & $94 \%$ & $6 \%$ \\
Resolver el ejercicio o problema con el estudiante & $97 \%$ & $3 \%$ \\
Repetir las propiedades de las operaciones & $79 \%$ & $21 \%$ \\
Dejar una guía de ejercicios o problemas adicional & $68 \%$ & $32 \%$ \\
Colocar otro problema/ejercicio para que sea resuelto por los estudiantes en la & $94 \%$ & $6 \%$ \\
pizarra & \\
\hline
\end{tabular}

Fuente: elaboración propia 
Los docentes aseguran, en más del 90\%, que utilizan la resolución de problemas en la pizarra, resolver un problema con los estudiantes y la propuesta de resolución de problemas de fracciones frente a la pizarra. Las menos seleccionadas fueron repetir las propiedades y la guía de ejercicios. En busca de profundizar en estas respuestas, en el cuadro J, se presentan las categorías construidas a partir de la pregunta ¿qué estrategias ha utilizado usted para enseñar las operaciones básicas con las fracciones? girada a los docentes (Cuadro 9).

Cuadro 9. Categorización de las estrategias docentes para la enseñanza de las OBF.

\begin{tabular}{|l|}
\hline Recursos manipulables \\
\hline Uso de representaciones geométricas \\
\hline Recursos digitales \\
\hline Juegos \\
\hline Trabajo tradicional \\
\hline Trabajo teórico \\
\hline Trabajo con ejercicios \\
\hline Retroalimentación clases \\
\hline
\end{tabular}

Fuente: Elaboración propia.

La categoría recursos manipulables recoge una diversa cantidad de estrategias utilizadas por los docentes. Esta estrategia está vinculada a la categoría representaciones geométricas por la que los docentes pretenden "hacer una demostración gráfica de las operaciones". Otras estrategias utilizadas son el uso de juegos y recursos digitales, es decir, softwares.

A pesar de estas estrategias y recursos aún se evidencian las llamadas metodologías tradicionales, para el caso, un docente consultado que inicia con "ejemplos sencillos hasta que se apropien el algoritmo de cada operación y luego ir aumentando la dificultad". Otro docente utiliza más el trabajo colaborativo entre estudiantes y la estrategia de retroalimentar clases es igualmente utilizada por un docente con el objetivo de afianzar los conocimientos del estudiantado.

El replanteamiento de la enseñanza de la matemática a una forma no tradicional no es un debate reciente. Para Lopes (2008), inspirado en un texto de Peter Hilton, la enseñanza de las fracciones debe ser realizada más allá de la forma tradicional a tal punto de reconsiderar el sentido o la pertinencia curricular del estudio de las fracciones si esta es enseñada como hasta 
hoy. El debate va, incluso, a si estas deben ser enseñadas como tema con espacio curricular definido, tal como lo plantea Carlos Vianna citado por Lopes (2008, p. 3)

Ahora bien, ¿A qué se debe esta posición crítica de la forma en que se ha enseñado las fracciones en la escuela? Para Lopes (2008, p. 4), citando a Hilton, esto se debe a que la enseñanza de las fracciones en la actualidad presenta cinco defectos curriculares: aplicaciones engañosas; confusión con la función de los decimales; ausencia de cuidados con definiciones y explicaciones; deshonestidad de la presentación y; la pasión por la ortodoxia.

De este modo, las fracciones parecen tener sentido únicamente en las matemáticas escolares para hacer operaciones. ¿Qué tanto de esto se ha perdido con el contexto social de la matemática? Lopes (2008) nos recuerda que el uso de las fracciones en el día a día se limita al trabajo de fracciones con medios, tercios, cuartos y doceavos, lejos de aquellos problemas que se pueden encontrar hoy en día en textos escolares donde se colocan ejercicios de fracciones extremadamente complejas. Ese abuso de lo que podríamos llamar hiperescolarización matemática fue llamado por Chevallard, Bosh y Gascón (1997) de enfermedad didáctica y es ejemplificado en Rolkouski y Vianna (2015).

Para Lopes la discusión acerca de cómo enseñar fracciones debe superar los problemas de contar partes de pizzas y chocolates a darle una mayor importancia a la complejidad de estos conceptos. En primer lugar, Lopes (2008) propone evitar las prisas por las definiciones, prisas que en las que se enseñan las fracciones de forma natural de la forma "parte/todo" e inmediatamente son trabajadas como parte del conjunto de los números racionales con todas las implicaciones cognitivas que esto lleva. Otro punto señalado por Lopes (2008) es ese paso natural que obvia que la nomenclatura de las fracciones es un obstáculo que enfrentan los estudiantes para la comprensión de este nuevo conjunto numérico.

Otro problema a considerar, en relación a la enseñanza de las fracciones que señala Lopes (2008, p.10-11), es la que parece ser una implícita "reserva de mercado" que tiene el tema en el currículo donde, a partir de un grado escolar determinado, estas dejan de ser enseñadas y para él:

Detrás de esta visión, subyace la creencia en el carácter categórico y acumulativo de los contenidos, basta enseñar fracciones en algún punto del programa y ¡listo! De allí en adelante las fracciones estarían disponibles como objeto de dominio de los alumnos. (Lopes, 2008, p. 10) 
Para Lopes este es un error que es confirmado en los años superiores cuando los docentes muestran su "incredulidad por el hecho de que sus alumnos no responden a actividades que envuelven fracciones con el desempeño esperado" (Lopes, 2008, p. 11). Esta situación podría mejorarse si en el resto del programa escolar se contemplaran experiencias con fracciones en todo nivel, eso sí, más allá de complejizarlas poniendo fracciones más difíciles (Lopes, 2008).

Ahora bien, ¿cómo creen los docentes que debe enfrentarse los errores que cometen los estudiantes al resolver operaciones básicas con fracciones? En un primer momento se les consultó qué tan efectivas consideraban que eran unas estrategias comunes para el tratamiento de las fracciones. Al consultarles al respecto, las respuestas fueron las siguientes, (Tabla 4).

Tabla 4. Estrategia es efectiva para tratar el error

\begin{tabular}{|c|c|c|}
\hline Estrategia & Sí & No \\
\hline Resuelve el ejercicio o problema en la pizarra & $97,1 \%$ & $2,9 \%$ \\
\hline Resuelve el ejercicio o problema con el estudiante & $94,1 \%$ & $5,9 \%$ \\
\hline Repetir las propiedades de las operaciones & $73,5 \%$ & $26,5 \%$ \\
\hline Dejar una guía de ejercicios o problemas adicional & $76,5 \%$ & $23,5 \%$ \\
\hline $\begin{array}{l}\text { Colocar otro problema/ejercicio para que sea resuelto por los estudiantes en } \\
\text { la pizarra }\end{array}$ & $97,1 \%$ & $2,9 \%$ \\
\hline
\end{tabular}

\section{Fuente: elaboración propia}

Los datos reflejan que las estrategias que son consideraras menos efectivas por los docentes son las mismas que eran las menos utilizadas por el magisterio. Ahora bien, para profundizar en estos elementos se les consultó a los docentes qué estrategias ellos utilizan en el tratamiento al error de los estudiantes al resolver OBF. Estas categorías, usando el AdC, se presentan en el Cuadro 10 siguiente.

Cuadro 10. Categorización de las estrategias docentes para el tratamiento de los errores de los estudiantes al resolver OBF.

Refuerzo de las operaciones

Refuerzo teórico

Trabajo grupal de estudiantes

Trabajo individual de estudiantes

Recursos manipulables

Recurso multimedia

Juegos

Fuente: Elaboración propia.

Las principales estrategias para el tratamiento de los errores son el refuerzo teórico y práctico de las $\mathrm{OBF}$. Al aplicar un refuerzo práctico el docente lo que pretende es ayudar a 
"reforzar los pasos con mayor dificultad, explicar más y hacer más ejercicios"; con el refuerzo teórico el docente pretende "explicar nuevamente los procedimientos. Partir de lo más fácil a lo complejo". El trabajo colaborativo también es una estrategia usada por los docentes, y en ella se involucra a los llamados tutores académicos que son otros estudiantes, del mismo grupo o no, con un mejor rendimiento académico.

Por último, los docentes informaron que para el tratamiento del error hacen uso de materiales manipulables, herramientas tecnológicas y juegos. El geoblocks es uno de los recursos manipulables que fueron mencionados, bingo de fracciones y plastilina de igual forma. El uso que le dan a estos materiales es como elementos de apoyo visual y a modo de fortalecer la comprensión teórica del tema. Los materiales audiovisuales son usados para que ellos puedan verificar procesos de resolución de problemas, aquí son mencionadas muchas redes sociales. El uso del juego en las fracciones es para incentivar al estudiante y que se interese en el tema.

Reconociendo que no existe un método único e infalible para la enseñanza de cualquier tema en cualquier área del conocimiento, incluyendo la matemática y la enseñanza de fracciones Lopes (2008) propone que los estudiantes sean expuestos a la problematización y exploración de la noción de mitad en distintos contextos de comparación; que investiguen el sentido de las palabras que tienen la idea de mitad, tercios, cuartos, quintos, doceavos, etc.; que explicite el sentido de las palabras relacionadas a la palabra "fracción": fragmento, fractal, fractura, frágil, fracaso, fraccionado, etc.; que explore la resolución de problemas que involucren la visualización; que trabaje con las fracciones egipcias ; la exploración y trabajo con fracciones "buenas", es decir, "fracciones que podemos construir una imagen mental, o que tengan alto significado cultural y de uso" (Lopes, 2008, p. 15) y que el estudiante comprenda el sentido histórico de las fracciones y su origen; el estudio de las fracciones en área de la probabilidad.;

\section{Conclusiones}

Este artículo pretendió analizar las concepciones docentes salvadoreños que enseñan matemática acerca del error de los estudiantes al resolver OBF. En este estudio se verificó una fuerte concepción negativa del error, siendo este poco valorado en el aula y sus causas están, según ellos mismos, en los descuidos o desinterés del estudiante y de malas prácticas docentes.

Este estudio también pretendió conocer las OBF que eran consideradas por los docentes como la más fácil y la más difícil. La multiplicación, con gran recurrencia, fue considerada por los docentes consultados como la operación básica con fracciones que resulta más fácil de 
resolver, en contraste la operación suma y resta destacaron como las más difíciles. Esto se debe, según el análisis, debido a la complejidad del algoritmo de la suma y resta, contrastando con la facilidad del algoritmo de la multiplicación.

Sobre las formas de enseñar las fracciones y el tratamiento del error, se pudo observar una predisposición de algunos docentes a metodologías menos tradicionalistas, sin embargo, no se nota claridad en cómo abordarlas. El refuerzo académico es una estrategia de tratamiento del error muy impregnado en las concepciones docentes. Este artículo quiso traer en ambos casos otros caminos y otras propuestas acerca de la enseñanza de las fracciones y del tratamiento positivo del error en el aula.

Se espera que en próximos estudios se pueda explorar las concepciones de los estudiantes salvadoreños y contrastar algunas conclusiones surgidas en este estudio.

\section{Referencias}

Abrate, R; Pochulu, M. y Vargas, J. (2006). Errores y dificultades en Matemática. Análisis de causas y sugerencias de trabajo $1^{\mathrm{a}}$ ed. Buenos Aires: Universidad Nacional de Villa María.

Astolfi, J.P. (2003). El error, un medio para enseñar 2da Ed. México: Díada Editorial.

Bogan, R; Biklen, S. (1994). Investigação qualitativa em Educação. Porto: Porto Editora.

Borasi, R. (1985). Using errors as springboards for the learning of mathematics: an introduction. Focus on learning problems in mathematics, v. 7, n. 3-4, p. 1-14.

Chevallard, Y; Bosch, M; Gascón, J. (1997). Estudiar Matemáticas. El eslabón perdido entre la enseñanza y el aprendizaje. Barcelona: Edtorial Horsori.

Creswell, J. W. (2010). Projeto de pesquisa, métodos qualitativos, quantitativo e misto. Porto Alegre: Artmed.

Cury, H. N. (2017). Análise de erros: o que podemos aprender com as respostas dos alunos, 2Ed. Autêntica Editores: Belo Horizonte.

Imbernón, F. (2001). Claves para una nueva formación del profesorado. Investigación en la Escuela. España, 57-66.

Lopes, A. J. (2008). O que Nossos Alunos Podem Estar Deixando de Aprender sobre Frações, quando tentamos lhes Ensinar Frações. Boletim de Educação Matemática, vol. 21, núm. 31, pp. 1-22.

Flores Peñafiel, A. (2014). División de fracciones como comparación multiplicativa a partir de métodos de los alumnos. Educación Matemática, 227-242. Recuperado 6 Julio 2020 de http://www.revista-educacion-matematica.org.mx/descargas/Esp-1-11.pdf.

Garnica, A. V. M. (2004). História Oral e Educação Matemática. In: BORBA, M. C.; ARAÚJO, J. L. (Org.) Pesquisa Qualitativa em Educação Matemática. Belo Horizonte: Autêntica, 2004. 
Rico, L. (1995). Errores y Dificultades en el Aprendizaje de las Matemáticas.

Rolkouski, E; Vianna, C.R. (2015). Maldades na prática com a matemática escolar. In. Kalinke, M.A; Mocrosky, L.F. (2015). Educação Matemática: pesquisas e possibilidades. Curitiba: Editora UFPR.

Sampieri, R. H.; Collado, C. F.; y Lucio, M. P. (2010). Metodología de la investigación. 5ta Edición. México: Mc Graw Hill.

Socas, M.M. (1997). Capítulo V: Dificultades, obstáculos y errores en el aprendizaje de las Matemáticas en la Educación Secundaria. En Rico, L. y otros: La Educación Matemática en la Enseñanza Secundaria. Barcelona: Horsori. Disponible en: https://laurabrichetti.files.wordpress.com/2010/12/socas-robayna-dificutades-errores-yobstc3a1culos-en-el-azaje-de-la-matemc3a1tica.pdf

Moraes, R. (1999). Análise de conteúdo. Revista Educação, Porto Alegre, v. 22, n. 37, p. 7-32.

Muniz, C. A. (2017). O ser matemático nos anos inicias e as produções subjetivas nas aprendizagens matemáticas: aprendizagem e diversidade. Encontro paranaense de Educação Matemática. Disponible en: http://www.sbemparana.com.br/eventos/index.php/EPREM/XIV_EPREM/paper/viewFile $\underline{1333 / 149}$

Ponte, J. P. (1992). Concepções dos Professores de Matemática e Processos de Formação. Disponible en: https://core.ac.uk/download/pdf/12423096.pdf.

\section{Autor}

Jeser Caleb Candray Menjivar, Investigador asociado al Instituto de Ciencia, Tecnología e Innovación, Universidad Francisco Gavidia, El Salvador. Doctorando en Educación Matemática por la Universidade Estadual Paulista "Júlio de Mesquita Filho" - Rio Claro, São Paulo, Brasil. Máster en Educación en Ciencia y en Matemática por la Universidade Federal do Paraná - Curitiba, Paraná, Brasil. Profesor y licenciado por la Universidad de El Salvador, El Salvador. Correo: jeser.candray@unesp.br// jccandray@hotmail.com. 